\title{
Reducción broncoscópica del volumen pulmonar mediante una válvula endobronquial para tratar una bulla enfisematosa extensa: reporte de un caso
}

\author{
Chin Kook Rhee Eung Gu Lee \\ División de Medicina Pulmonar, de Alergias y Cuidados Intensivos, Departamento de Medicina Interna, Hospital St. Mary de Seúl, \\ Colegio de Medicina, Universidad Católica de Corea, Seúl, República de Corea
}

\begin{abstract}
Palabras clave
Enfermedad pulmonar obstructiva crónica (EPOC) · Bulla . Reducción broncoscópica del volumen pulmonar (RBVP) . Válvula endobronquial (VEB)
\end{abstract}

\section{Resumen}

Antecedentes: En pacientes con enfermedad pulmonar obstructiva crónica (EPOC), las técnicas de reducción broncoscópica del volumen pulmonar (RBVP) que emplean válvulas endobronquiales unidireccionales mejoran la función pulmonar y aumentan la tolerancia al ejercicio. El tratamiento de RBVP está incluido en los lineamientos terapéuticos de la Iniciativa Global para la Enfermedad Pulmonar Obstructiva Crónica (GOLD) para pacientes con EPOC sin ventilación colateral interlobular. Sin embargo, no se ha intentado la RBVP con válvula endobronquial en pacientes con bulla gigante. Presentación del caso: Reportamos una RBVP exitosa y segura utilizando una válvula endobronquial en un paciente con un enfisema bulloso extenso en el lóbulo medio derecho. Un varón de 65 años fue diagnosticado con EPOC 5 años antes, y al momento presentaba una bulla de gran tamaño en el lóbulo medio derecho.
Durante el seguimiento regular, los síntomas de dificultad para respirar empeoraron gradualmente, y el tamaño de la bulla se incrementó gradualmente de acuerdo con la tomografía computarizada (TC). Por tanto, decidimos tratar al paciente con RBVP mediante una válvula endobronquial unidireccional. El sistema Chartis (Pulmonx, Inc., Palo Alto, CA, EE. UU.) confirmó la ausencia de ventilación colateral en el bronquio medio derecho. Insertamos exitosamente una válvula endobronquial en el bronquio medio derecho. Tras la inserción, la bulla disminuyó drásticamente su tamaño, y los síntomas del paciente y su calidad de vida mejoraron marcadamente.

Conclusión: Este caso apoya la reciente sugerencia de que la RBVP puede ser un tratamiento alternativo exitoso para pacientes seleccionados apropiadamente.

(c) $2019 \mathrm{El} /$ Los autor(es)

\section{Antecedentes}

La enfermedad pulmonar obstructiva crónica (EPOC) se caracteriza por síntomas respiratorios persistentes y limitaciones al flujo de aire causadas por la combinación de reducción patológica de las vías respiratorias (bronquiolitis crónica) y la destrucción del parénquima (enfisema) [1]; este último involucra un crecimiento permanente del espacio aéreo en las partes distales de los bron- quiolos terminales debido a la destrucción de los sacos alveolares. El enfisema desencadena la pérdida de tejido elástico, el colapso de las vías respiratorias y dificultades para el intercambio gaseoso [2].

Entre los tratamientos convencionales para la EPOC se encuentran el abandono del tabaquismo, fármacos inhalados como beta-agonistas de acción prolongada y agentes antimuscarínicos, y oxigenoterapia de largo plazo. La cirugía de reducción de volu- information@karger.com

() 2020 S.Karger GmbH, Freiburg

www.karger.com $/ \mathrm{kxn}$

Karger ${ }^{\prime \prime}=$
Prof. Chin Kook Rhee, M.D., Ph.D.

División de Medicina Pulmonar, de Alergias y Cuidados Intensivos Departamento de Medicina Interna

Hospital St. Mary de Seúl, Colegio de Medicina, Universidad Católica de Corea 222 Banpo-daero, Seocho-gu, Seúl, 06591, República de Corea chinkook77@gmail.com 
men pulmonar (CRVP) mejora la tasa de supervivencia de los pacientes con enfisema predominante del lóbulo superior y baja capacidad para el ejercicio [1]. En tales pacientes, la CRVP ofrece mayores beneficios de supervivencia que otros tratamientos médicos. Sin embargo, el enfisema sin predominancia en los lóbulos superiores es el único predictor de mortalidad operativa [3].

En pacientes seleccionados con función pulmonar disminuida, enfisema avanzado resistente a la terapia o incapacidad de someterse a CRVP, la reducción broncoscópica del volumen pulmonar (RBVP) empleando una válvula endobronquial unidireccional mejora la tolerancia al ejercicio y la función pulmonar $[4,5]$. La implantación de la válvula impide la hiperinflación, al bloquear el flujo de aire inspiratorio al pulmón intervenido, y permite que el aire escape durante la exhalación. Al reducir el tamaño de un pulmón enfisematoso hiperexpandido, el pulmón restante se reexpande, y la función pulmonar total mejora [6]. El tratamiento con RBVP está incluido en los lineamientos terapéuticos de la Iniciativa Global para la Enfermedad Pulmonar Obstructiva Crónica (Global Initiative for Chronic Obstructive Lung Disease, GOLD) para pacientes con EPOC sin ventilación colateral interlobular. Park et al. estudiaron los resultados a un largo plazo relativo de RBVP con colocación de válvula endobronquial en pacientes coreanos con enfisema grave; se demostró que el procedimiento es seguro y efectivo [7]. Sin embargo, se excluyó a pacientes con bullas gigantes (más de $5 \mathrm{~cm}$ de diámetro); no se ha reportado un intento de usar RBVP/colocación de válvula endobronquial para tratar pacientes con bulla gigante. En este trabajo reportamos una RBVP exitosa y segura usando una válvula endobronquial en un paciente con enfisema bulloso extenso en el lóbulo medio derecho.

\section{Presentación del caso}

Un varón de 65 años diagnosticado con EPOC 5 años antes fue ingresado en nuestro hospital en noviembre de 2017. El paciente había dejado de fumar 2 años antes, pero tenía una historia de tabaquismo de 80 paquetes-año. Había estado recibiendo indacate$\mathrm{rol} /$ glicopirronio una vez al día y recibió $3.5 \mathrm{~L} / \mathrm{min}$ de oxigenoterapia en casa por 2 años. En el año anterior había experimentado dos exacerbaciones agudas que requirieron hospitalización. Una prueba de función pulmonar (PFP) realizada en octubre de 2017 reveló una enfermedad pulmonar obstructiva grave: la razón de volumen espiratorio forzado en $1 \mathrm{~s}\left(\mathrm{VEF}_{1}\right)$ con respecto a la capacidad vital forzada (CVF) fue $29 \%$, el $\mathrm{VEF}_{1}$ fue $0.41 \mathrm{~L}$ (porcentaje de $\mathrm{VEF}_{1}$ predicho, 13\%), el volumen residual (VR) fue $6.43 \mathrm{~L}$ (porcentaje de VR predicho, 275\%); la capacidad pulmonar total (CPT) fue $8.23 \mathrm{~L}$ (porcentaje de CPT predicha, 135\%), y el porcentaje de capacidad de difusión de monóxido de carbono $\left(\mathrm{D}_{\mathrm{LCO}}\right)$ fue $23 \%$. $\mathrm{El}$ análisis de gases de la sangre arterial reveló un $\mathrm{pH}$ de 7.413, $\mathrm{P}_{\mathrm{a}} \mathrm{CO}_{2}$ de $53.8 \mathrm{mmHg}$ y $\mathrm{P}_{\mathrm{a}} \mathrm{O}_{2}$ de $65.4 \mathrm{mmHg}$.

Una tomografía computarizada (TC) del pecho practicada en mayo de 2017 indicó enfisema centrolobulillar en ambos pulmones, con una bulla extensa en el lóbulo medio derecho (Fig. 1c y e). El área máxima de la bulla en la vista axial fue $15.0 \times 10.1 \mathrm{~cm}$.
De acuerdo con la TC, la bulla creció con el tiempo y el parénquima del lóbulo inferior derecho sufrió una compresión cada vez mayor. La fisura alrededor del lóbulo medio derecho (el blanco terapéutico) aparecía intacta en la TC del pecho.

Decidimos realizar una RBVP empleando una válvula endobronquial unidireccional. Se administraron $0.5 \mathrm{mg}$ de atropina $30 \mathrm{~min}$ antes de la broncoscopía, para minimizar las secreciones bronquiales. Para anestesiar localmente la orofaringe, se administraron $2 \mathrm{~mL}$ de lidocaína mediante un nebulizador. Al inicio de la broncoscopía se administraron $3 \mathrm{mg}$ de midazolam para provocar sedación y se instilaron $10 \mathrm{~mL}$ de lidocaína para anestesiar localmente las cuerdas vocales y las vías respiratorias mayores. Empleando el sistema Chartis (Pulmonx, Inc., Palo Alto, CA, EE. UU.), un catéter con un globo en la punta distal se colocó en la entrada del bronquio medio derecho. El globo se infló para bloquear la vía respiratoria y se midieron el flujo de aire, la resistencia y la presión distales. Se observó una disminución gradual en el flujo y un incremento en la resistencia y la presión. Así, confirmamos la ausencia de ventilación colateral en el lóbulo medio derecho [8]. El bronquio medio derecho fue lo suficientemente estrecho para su bloqueo con una sola válvula endobronquial; para lograrlo, seleccionamos una válvula endobronquial Zephyr 5.5 (Pulmonx).

Luego de la inserción de la válvula endobronquial, se tomaron placas de rayos $\mathrm{X}$ del pecho por 3 días y una semana después, y se confirmó que el tamaño de la bulla había disminuido dramáticamente (Fig. 1a y b). Dos meses después de la inserción de la válvula se practicó una TC del pecho, que mostró que la válvula se localizaba en el bronquio medio derecho, y que la extensa bulla en el lóbulo medio derecho había desaparecido. De este modo, el volumen del lóbulo medio derecho había decrecido, pero el del lóbulo inferior derecho previamente comprimido se había reexpandido (Fig. 1d y f).

Realizamos una PFP 2 meses después de la inserción de la válvula. La razón de $\mathrm{VEF}_{1}$ con respecto a CVF se incrementó de 29 a $32 \%$, y el $\mathrm{VEF}_{1}$ aumentó en $170 \mathrm{~mL}$, de $0.41 \mathrm{~L}$ (porcentaje de $\mathrm{VEF}_{1}$ predicho, 13\%) a $0.58 \mathrm{~L}$ (19\%). El VR disminuyó de $6.43 \mathrm{~L}$ (porcentaje de VR predicho, 275\%) a 4.74 L (201\%), y la CPT disminuyó de 8.23 L (porcentaje de CPT predicha, 135\%) a $6.61 \mathrm{~L}(108 \%)$. Los síntomas y la calidad de vida del paciente mejoraron marcadamente, y hasta la fecha no se ha observado migración u obstrucción de la válvula, neumonía o neumotórax.

\section{Discusión}

Convencionalmente, se considera que la fisiopatología del enfisema bulloso involucra una obstrucción valvular que permite al aire entrar, pero no salir del espacio quístico. Sin embargo, una nueva hipótesis sugiere que el enfisema bulloso está asociado con la comunicación de la vía respiratoria libre causada por una compliancia mayor que la del pulmón alrededor [9]. Para las bullas que no son alimentadas por una vía respiratoria, el tratamiento con válvula endobronquial probablemente no será efectivo. Sin embargo, en el presente caso, el tamaño de la bulla se incrementó con el 
Fig. 1. (a), (c) y (e) Los rayos $X$ del pecho tomados antes del procedimiento (noviembre de 2017) y la tomografía computarizada (TC) tomada en mayo de 2017 (vistas transversal y sagital, respectivamente) indicaron enfisema grave y una bulla extensa en el lóbulo medio derecho que comprimía el parénquima del lóbulo inferior derecho. (b), (d) y (f) La placa de rayos $X$ del pecho tomada una semana después del procedimiento (noviembre de 2017) y la TC tomada 2 meses después del procedimiento (enero de 2018, vista transversal y sagital, respectivamente) mostraron que la extensa bulla en el lóbulo medio derecho había desaparecido. Esto causó una reducción en el volumen del lóbulo medio derecho, mientras el lóbulo inferior derecho, antes comprimido, se reexpandió.
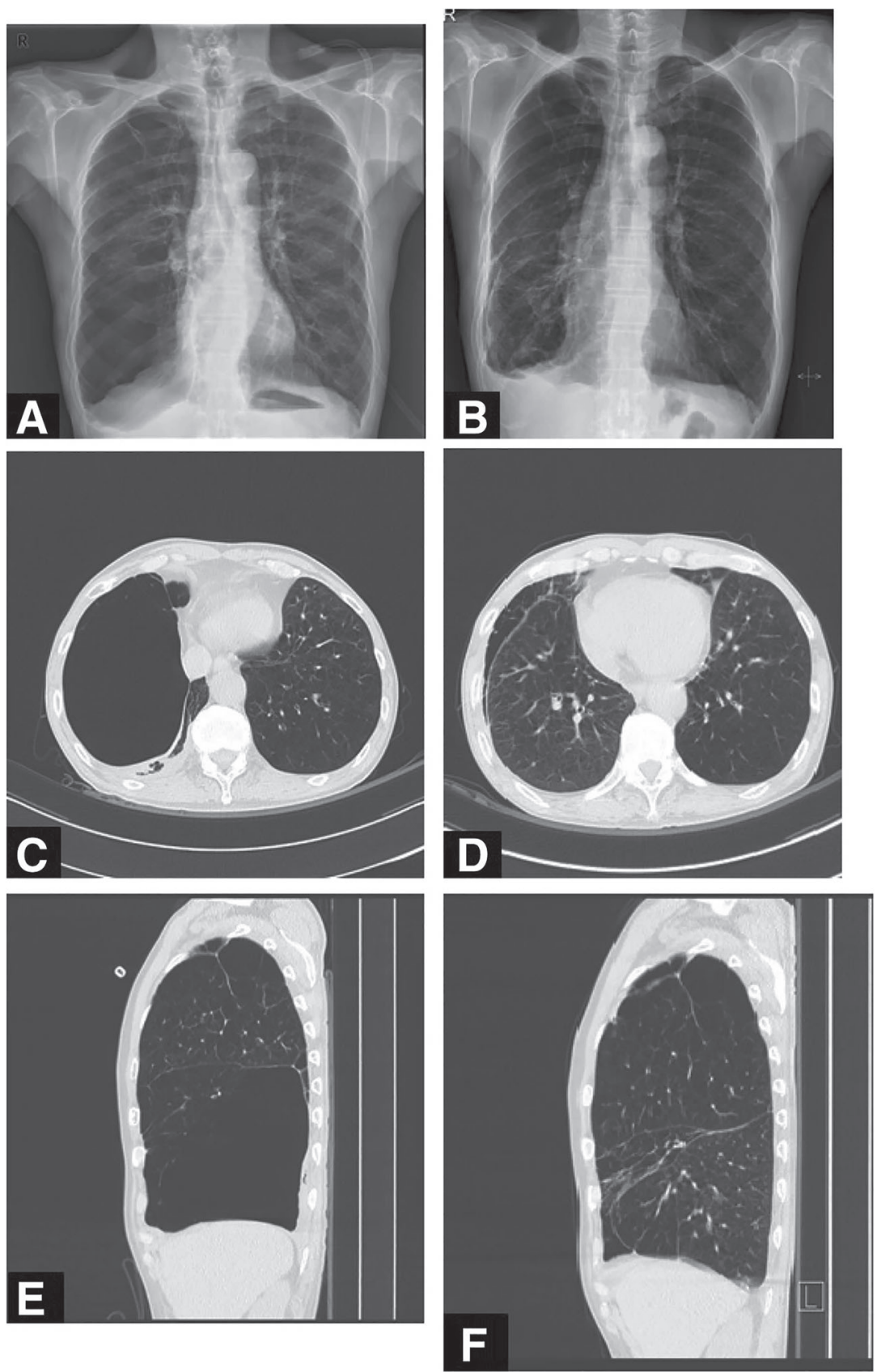

tiempo, lo que indica su comunicación con la vía respiratoria. Así, cuando las bullas son extensas, contenidas en un lóbulo y muy probablemente alimentadas por una vía respiratoria, la reducción del volumen lobular y la reducción del volumen de la bulla son esencialmente idénticas (es decir, el mecanismo/objetivo es el mismo).

Aunque no se han documentado diferencias en la tasa de supervivencia o resultados a largo plazo entre pacientes que reciben RBVP y CRVP, la RBVP parece reducir efectivamente la mortalidad y mejora la calidad de vida de pacientes que no responden al tratamiento médico y presentan alto riesgo de complicaciones quirúrgicas [5]. Klooster et al. encontraron que la colocación de una válvula endobronquial en pacientes seleccionados apropiadamente mejoró de manera significativa la función pulmonar y la capacidad para el ejercicio [5]. Para optimizar el resultado clínico, se requieren criterios de selección estrictos al identificar a pacientes que pueden beneficiarse con la RBVP [10]. Flandes et al. propusieron los siguientes criterios: EPOC sintomática; una puntuación de disnea $>1$ en la escala modificada Medical Research Council (mMRC); fenotipo predominante de enfisema; no más de dos exacerbaciones anuales de EPOC; obstrucción grave del flujo de aire; hiperinflación y captura de aire evidente en la PFP; sin fumar 
por al menos 6 meses, ausencia de cirugía previa en el lado del lóbulo que se va a intervenir, y sin ventilación colateral según el sistema Chartis [11]. Nuestro paciente cumplió con todos estos criterios, así que realizamos la reducción de volumen pulmonar utilizando una válvula endobronquial luego de confirmar la ausencia de ventilación colateral. En todo el mundo han aparecido dos reportes de caso en donde se empleó la RBVP para tratar bullas gigantes en el lóbulo medio derecho [12, 13]. Nuestros resultados son superiores, ya que nuestro paciente tenía una función pulmonar basal menor que los pacientes en los reportes de caso citados.

\section{Conclusión}

Este estudio de caso apoya las sugerencias recientes de que la RBVP puede ser un buen tratamiento alternativo para pacientes seleccionados apropiadamente.

\section{Conflicto de interés}

Los autores declaran que no tienen conflicto de interés.

\section{Disponibilidad de datos y materiales}

Todos los datos relacionados con los hallazgos están disponibles dentro del manuscrito.

\section{Información de licencia}

Eung Gu Lee, Chin Kook Rhee: Reducción broncoscópica del volumen pulmonar mediante una válvula endobronquial para tratar una bulla enfisematosa extensa: reporte de un caso. BMC Pulm Med 2019;19:204 (https:// doi.org/10.1186/s12890-019-0849-z). ${ }^{\odot} \mathrm{El}$ (los) autor(es) 2019 (traducción, abreviaturas, agradecimientos, financiamiento, nota del editor acortada), licensiado bajo CC BY 4.0 (https://creativecommons.org/licenses/by/4.0/ deed.es)

\section{Referencias}

-1 Vogelmeier CF, Criner GJ, Martinez FJ, et al.: Global strategy for the diagnosis, management, and prevention of chronic obstructive lung disease 2017 report. GOLD executive summary. Am J Respir Crit Care Med 2017; 195(5):557-582.

2 Kemp SV, Polkey MI, Shah PL: The epidemiology, etiology, clinical features, and natural history of emphysema. Thorac Surg Clin 2009; 19(2):149-158.

-3 Criner GJ: Alternatives to lung transplantation: lung volume reduction for COPD. Clin Chest Med 2011;32(2):379-397.

4 Valipour A, Slebos DJ, Herth F, et al.: Endobronchial valve therapy in patients with homogeneous emphysema. Results from the IMPACT Study. Am J Respir Crit Care Med 2016; 194(9):1073-1082.
5 Klooster K, Slebos DJ, Zoumot Z,et al.: Endobronchial valves for emphysema: an individual patient-level reanalysis of randomised controlled trials. BMJ Open Respir Res 2017; 4(1):e000214.

6 Valipour A: Valve therapy in patients with emphysematous type of chronic obstructive pulmonary disease (COPD): from randomized trials to patient selection in clinical practice. J Thorac Dis 2018;10(Suppl 23):S2780-2796.

$\checkmark 7$ Park TS, Hong Y, Lee JS, Oet al.: Bronchoscopic lung volume reduction by endobronchial valve in advanced emphysema: the first Asian report. Int J Chron Obstruct Pulmon Dis 2015; 10:1501-1511.

8 Davey C, Zoumot Z, Jordan S, et al.: Bronchoscopic lung volume reduction with endobronchial valves for patients with heterogeneous emphysema and intact interlobar fissures (the BeLieVeR-HIFi study): a randomised controlled trial. Lancet 2015;386(9998):10661073.
9 Klingman RR, Angelillo VA, DeMeester TR: Cystic and bullous lung disease. Ann Thorac Surg 1991;52(3):576-580.

10 Aggelou K, Siafakas N: Medical lung volume reduction for severe emphysema: a review. Respir Med 2017;131:141-147.

11 Flandes J, Soto FJ, Cordovilla R, et al.: Bronchoscopic lung volume reduction. Clin Chest Med 2018;39(1):169-180.

12 Tian Q, An Y, Xiao BB,et al.: Treatment of giant emphysamous bulla with endobronchial valves in patients with chronic obstructive pulmonary disease: a case series. J Thorac Dis 2014;6(12):1674-1680.

13 Hou G, Wang W, Wang QY,et al.: Bronchoscopic bullectomy with a one-way endobronchial valve to treat a giant bulla in an emphysematic lung: a case report. Clin Respir J 2016; 10(5):657-660. 